Bull. Austral. Math. Soc.

VoL. 66 (2002) [385-391]

\title{
ON BOUNDEDNESS OF THE WEIGHTED BERGMAN PROJECTIONS ON THE LIPSCHITZ SPACES
}

\author{
Hong Rae Cho and Jinkee Lee
}

In this paper we study the boundedness of the weighted Bergman projections on the weighted subspaces of Bergman spaces and the Lipschitz spaces on the unit ball and the unit polydisc.

\section{INTRODUCTION}

Let $B_{n}$ and $D^{n}$ be the unit ball and the unit polydisc in $\mathbb{C}^{n}$, respectively. Let $-1<\gamma<\infty$ and $0<p<\infty$. Let $L_{\gamma}^{p}\left(B_{n}\right)$ and $L_{\gamma}^{p}\left(D^{n}\right)$ be $L^{p}$-spaces with respect to the weighted volume measures

$$
d V_{\gamma}(z)=\left(1-|z|^{2}\right)^{\gamma} d V(z), \quad \prod_{j=1}^{n}\left(1-\left|z_{j}\right|^{2}\right)^{\gamma} d V(z)
$$

on $B_{n}$ and $D^{n}$, respectively. Let $A_{\gamma}^{p}\left(B_{n}\right)$ and $A_{\gamma}^{p}\left(D^{n}\right)$ be subspaces of $L_{\gamma}^{p}\left(B_{n}\right)$ and $L_{\gamma}^{p}\left(D^{n}\right)$ consisting of functions which are holomorphic on $B_{n}$ and $D^{n}$, respectively. They are called the weighted Bergman spaces. We define

$$
P_{\gamma} f(z)=C_{n, \gamma} \int_{B_{n}} \frac{f(\zeta)}{(1-\bar{\zeta} \cdot z)^{n+1+\gamma}}\left(1-|\zeta|^{2}\right)^{\gamma} d V(\zeta), \quad z \in B_{n}
$$

where

$$
C_{n, \gamma}=\frac{n !}{\pi^{n}} \frac{\Gamma(n+1+\gamma)}{\Gamma(n+1) \Gamma(\gamma+1)} .
$$

For the unit polydisc we define

$$
P_{\gamma} f(z)=C_{n, \gamma} \int_{D^{n}} f(\zeta) \prod_{j=1}^{n} \frac{\left(1-\left|\zeta_{j}\right|^{2}\right)^{\gamma}}{\left(1-\overline{\zeta_{j}} z_{j}\right)^{\gamma+2}} d V(\zeta), \quad z \in D^{n}
$$

Received 5th February, 2002

The first author was supported by grant No. R01-2000-00001 from the Basic Research Program of the Korea Science and Engineering Foundation and the second author was supported by the Brain Korea 21 project, 2001

Copyright Clearance Centre, Inc. Serial-fee code: 0004-9727/02 $\$ A 2.00+0.00$. 
where

$$
C_{n, \gamma}=\left(\frac{\gamma+1}{\pi}\right)^{n}
$$

They are orthogonal projections on $L_{\gamma}^{2}\left(B_{n}\right)$ and $L_{\gamma}^{2}\left(D^{n}\right)$ onto $A_{\gamma}^{2}\left(B_{n}\right)$ and $A_{\gamma}^{2}\left(D^{n}\right)$, respectively. They are called the weighted Bergman projections on $B_{n}$ and $D^{n}$, respectively.

In this paper we study the boundedness of the weighted Bergman projections on the weighted subspaces of Bergman spaces and the Lipschitz spaces.

\section{2. $L_{\gamma}^{p, \alpha}$ BOUNDEDNESS}

For $0<p<\infty,-1<\gamma<\infty$ and $\alpha>0, L_{\gamma}^{p, \alpha}\left(B_{n}\right)$ is defined to be the class of those $f \in L_{\gamma}^{p}\left(B_{n}\right)$ for which

$$
\sup _{z \in B_{n}}|f(z)|\left(1-|z|^{2}\right)^{\alpha}<\infty
$$

For $f \in L_{\gamma}^{p, \alpha}\left(B_{n}\right)$, we define

$$
\|f\|_{L_{\gamma}^{p, \alpha}\left(B_{n}\right)}:=\max \left(\|f\|_{L_{\gamma}^{p}\left(B_{n}\right)}, \sup _{z \in B_{n}}|f(z)|\left(1-|z|^{2}\right)^{\alpha}\right) .
$$

Then the weighted subspace $L_{\gamma}^{p, \alpha}\left(B_{n}\right)$ of $L_{\gamma}^{p}\left(B_{n}\right)$ is a Banach space with the norm $\|\cdot\|_{L_{\gamma}^{p, \alpha}\left(B_{n}\right)}$ when $1 \leqslant p<\infty$. Let $A_{\gamma}^{p, \alpha}\left(B_{n}\right)$ be the subspace of $L_{\gamma}^{p, \alpha}\left(B_{n}\right)$ consisting of functions which are holomorphic on $B_{n}$. We note that

$$
\|f\|_{L_{\gamma}^{p}\left(B_{n}\right)}^{p} \leqslant\left(\sup _{z \in B_{n}}|f(z)|\left(1-|z|^{2}\right)^{\alpha}\right)^{p} \int_{B_{n}}\left(1-|z|^{2}\right)^{\gamma-\alpha p} d V(z) .
$$

Thus for $f \in A_{\gamma}^{p, \alpha}\left(B_{n}\right)$ it follows that

$$
\|f\|_{L_{\gamma}^{p, \alpha}\left(B_{n}\right)} \approx \sup _{z \in B_{n}}|f(z)|\left(1-|z|^{2}\right)^{\alpha} \quad \text { for } \quad \alpha<\frac{(n+\gamma)}{p} .
$$

We can see that $([7,2])$ for $0<p<\infty$ and $-1<\gamma<\infty$

$$
f(z)=\mathcal{O}\left(\frac{1}{\left(1-|z|^{2}\right)^{(n+1+\gamma) / p}}\right) \text { for } f \in A_{\gamma}^{p}\left(B_{n}\right)
$$

Hence $A_{\gamma}^{p, \alpha}\left(B_{n}\right)=A_{\gamma}^{p}\left(B_{n}\right)$ for $\alpha \geqslant(n+1+\gamma) / p$.

For the polydisc we define $L_{\gamma}^{p, \alpha}\left(D^{n}\right)$ by the class of those $f \in L_{\gamma}^{p}\left(D^{n}\right)$ for which

$$
\sup _{z \in D^{n}}|f(z)| \prod_{j=1}^{n}\left(1-\left|z_{j}\right|^{2}\right)^{\alpha}<\infty
$$


For $f \in L_{\gamma}^{p, \alpha}\left(D^{n}\right)$, we define

$$
\|f\|_{L_{\gamma}^{p, \alpha}\left(D^{n}\right)}:=\max \left(\|f\|_{L_{\gamma}^{p}\left(D^{n}\right)}, \sup _{z \in D^{n}}|f(z)| \prod_{j=1}^{n}\left(1-\left|z_{j}\right|^{2}\right)^{\alpha}\right) .
$$

Let $A_{\gamma}^{p, \alpha}\left(D^{n}\right)$ be the subspace of $L_{\gamma}^{p, \alpha}\left(D^{n}\right)$ consisting of functions which are holomorphic on $D^{n}$. By the representation (1.2), Hölder's inequality, and (i) of Lemma 2.1, we can see that

$$
f(z)=\mathcal{O}\left(\frac{1}{\prod_{j=1}^{n}\left(1-\left|z_{j}\right|^{2}\right)^{(2+\gamma) / p}}\right) \quad \text { for } \quad f \in A_{\gamma}^{p}\left(D^{n}\right) .
$$

Hence $A_{\gamma}^{p, \alpha}\left(D^{n}\right)=A_{\gamma}^{p}\left(D^{n}\right)$ for $\alpha \geqslant(2+\gamma) / p$.

For an account of the known results on these spaces, see $[4,6]$.

Lemma 2.1. ([7]) For $z \in B_{n}, c$ real, $t>-1$, define

$$
J_{c, t}(z)=\int_{B_{n}} \frac{\left(1-|\zeta|^{2}\right)^{t}}{|1-\bar{\zeta} \cdot z|^{n+1+t+c}} d V(\zeta)
$$

where $d V(\zeta)$ is the volume measure.

(i) When $c>0$, then

$$
J_{c, t}(z) \approx\left(1-|z|^{2}\right)^{-c}
$$

(ii) When $c=0$, then

$$
J_{0, t}(z) \approx \log \frac{1}{1-|z|^{2}}
$$

The notation $a(z) \approx b(z)$ means that the ratio $a(z) / b(z)$ has a positive finite limit as $|z| \rightarrow 1$.

In [1] we can see that the weighted Bergman projection $P_{\gamma}$ maps $L_{\gamma}^{p}\left(B_{n}\right)$ onto $A_{\gamma}^{p}\left(B_{n}\right)$, boundedly, for $1<p<\infty$ and $\gamma>-1$. In this section we consider the boundedness of $P_{\gamma}$ on weighted subspaces $L_{\gamma}^{p, \alpha}$ of $L_{\gamma}^{p}$.

THEOREM 2.2. For $1 \leqslant p<\infty, \gamma>-1$, and $0<\alpha<\gamma+1$, the weighted Bergman projection $P_{\gamma}$ maps $L_{\gamma}^{p, \alpha}\left(B_{n}\right)$ onto $A_{\gamma}^{p, \alpha}\left(B_{n}\right)$, boundedly.

Proof: From (1.1) we have

$$
\begin{aligned}
\left|P_{\gamma} f(z)\right| & \lesssim \int_{B_{n}}|f(\zeta)| \frac{\left(1-|\zeta|^{2}\right)^{\gamma}}{|1-\bar{\zeta} \cdot z|^{n+1+\gamma}} d V(\zeta) \\
& \leqslant \sup _{\zeta \in B_{n}}|f(\zeta)|\left(1-|\zeta|^{2}\right)^{\alpha} \int_{B_{n}} \frac{\left(1-|\zeta|^{2}\right)^{\gamma-\alpha}}{|1-\bar{\zeta} \cdot z|^{n+1+\gamma}} d V(\zeta)
\end{aligned}
$$


By (i) of Lemma 2.1, the right side integral of the last inequality is bounded by $1 /(1$ $\left.-|z|^{2}\right)^{\alpha}$. Thus we have

$$
\left|P_{\gamma} f(z)\right|\left(1-|z|^{2}\right)^{\alpha} \lesssim \sup _{\zeta \in B_{n}}|f(\zeta)|\left(1-|\zeta|^{2}\right)^{\alpha}, \quad z \in B_{n}
$$

First we consider the case $1<p<\infty$. In [1] we can see that

$$
\left\|P_{\gamma} f\right\|_{L_{\gamma}^{p}\left(B_{n}\right)} \lesssim\|f\|_{L_{\gamma}^{p}\left(B_{n}\right)} \quad \text { for } \quad 1<p<\infty .
$$

By (2.1) and (2.2), we get the result for the case $1<p<\infty$.

Now we consider the case $p=1$. By (2.1), it follows that

$$
\begin{aligned}
\left\|P_{\gamma} f\right\|_{L_{\gamma}^{1}\left(B_{n}\right)} & =\int_{B_{n}}\left|P_{\gamma} f(z)\right|\left(1-|z|^{2}\right)^{\gamma} d V(z) \\
& \lesssim \int_{B_{n}}\left(1-|z|^{2}\right)^{\gamma-\alpha} d V(z) .
\end{aligned}
$$

Since $0<\alpha-\gamma<1$, the last integral is bounded by the constant depending on $\gamma, \alpha$, and $n$. By (2.1) and (2.3), we get the result for the case $p=1$. Therefore the result holds for all cases $1 \leqslant p<\infty$.

THEOREM 2.3. For $1 \leqslant p<\infty, \gamma>-1$, and $0<\alpha<\gamma+1$, the weighted Bergman projection $P_{\gamma}$ maps $L_{\gamma}^{p, \alpha}\left(D^{n}\right)$ onto $A_{\gamma}^{p, \alpha}\left(D^{n}\right)$, boundedly.

Proof: In [3] we can see that

$$
\left\|P_{\gamma} f\right\|_{L_{\gamma}^{p}\left(D^{n}\right)} \lesssim\|f\|_{L_{\gamma}^{p}\left(D^{n}\right)} \text { for } \quad 1<p<\infty
$$

By the similar method as the proof of Theorem 2.2, we can get the result.

\section{HÖLDER BOUNDEDNESS}

In order to prove that a function belongs to a Lipschitz space $\Lambda_{\alpha}$ we shall use the following Hardy-Littlewood type lemma.

Lemma 3.1. Let $\Omega \Subset \mathbb{C}^{n}$ be a domain with piecewise smooth boundary. Suppose $f \in C^{1}(\Omega)$ and that for some $0<\alpha<1$ there is a constant $C$, such that

$$
|\nabla f(z)| \leqslant C \delta_{\Omega}(z)^{\alpha-1} \text { for all } z \in \Omega,
$$

where $\delta_{\Omega}(z)$ is the distance function for $\Omega$. Then $f \in \Lambda_{\alpha}(\Omega)$.

The proof of the above lemma and of more general results about the Lipschitz spaces can be found in [5]. 
THEOREM 3.2. Suppose $0<\alpha<1$. Then the weighted Bergman projection $P_{\gamma}$ maps $\Lambda_{\alpha}\left(B_{n}\right)$ onto $\Lambda_{\alpha}\left(B_{n}\right)$, boundedly.

Proof: By symmetry, for $z=\left(z_{1}, \cdots, z_{n}\right) \in B_{n}$, it suffices to treat the case $j=1$, that is,

$$
\left|\frac{\partial}{\partial z_{1}} P_{\gamma} f(z)\right| \lesssim|f|_{\Lambda_{\alpha}\left(B_{n}\right)}\left(1-|z|^{2}\right)^{\alpha-1}
$$

By (1.1), we have

$$
\begin{aligned}
\frac{\partial}{\partial z_{1}} P_{\gamma} f(z)= & C_{n, \gamma} \int_{B_{n}} \frac{f(\zeta) \bar{\zeta}_{1}}{(1-\bar{\zeta} \cdot z)^{n+\gamma+2}}\left(1-|\zeta|^{2}\right)^{\gamma} d V(\zeta) \\
= & C_{n, \gamma} \int_{B_{n}} \frac{(f(\zeta)-f(z)) \bar{\zeta}_{1}}{(1-\bar{\zeta} \cdot z)^{n+\gamma+2}}\left(1-|\zeta|^{2}\right)^{\gamma} d V(\zeta) \\
& \quad+C_{n, \gamma} \int_{B_{n}} \frac{f(z) \bar{\zeta}_{1}}{(1-\bar{\zeta} \cdot z)^{n+\gamma+2}}\left(1-|\zeta|^{2}\right)^{\gamma} d V(\zeta) \\
= & I(z)+I I(z)
\end{aligned}
$$

Since

$$
C_{n, \gamma} \int_{B_{n}} \frac{1}{(1-\bar{\zeta} \cdot z)^{n+1+\gamma}}\left(1-|\zeta|^{2}\right)^{\gamma} d V(\zeta)=1,
$$

we have, by differentiating the integral above with respect to $z_{1}$,

$$
C_{n, \gamma} \int_{B_{n}} \frac{\bar{\zeta}_{1}}{(1-\bar{\zeta} \cdot z)^{n+2+\gamma}}\left(1-|\zeta|^{2}\right)^{\gamma} d V(\zeta)=0
$$

and we then have $I I(z)=0$.

Now $|\zeta-z| /|1-\bar{\zeta} \cdot z|<1$, and using the property (i) of Lemma 2.1, we have

$$
\begin{aligned}
|I(z)| & \lesssim \int_{B_{n}} \frac{|\zeta-z|^{\alpha}|f|_{\Lambda_{\alpha}}}{|1-\bar{\zeta} \cdot z|^{n+\gamma+2}}\left(1-|\zeta|^{2}\right)^{\gamma} d V(\zeta) \\
& \leqslant|f|_{\Lambda_{\alpha}\left(B_{n}\right)} \int_{B_{n}} \frac{\left(1-|\zeta|^{2}\right)^{\gamma}}{|1-\bar{\zeta} \cdot z|^{n+\gamma+2-\alpha}} d V(\zeta) \\
& \lesssim|f|_{\Lambda_{\alpha}\left(B_{n}\right)} \frac{1}{\left(1-|z|^{2}\right)^{1-\alpha}} .
\end{aligned}
$$

Thus we get (3.1).

We consider the case of the unit polydisc, it can be treated in the same way as in the proof of Theorem 3.2. 
Theorem 3.3. Suppose $0<\beta<\alpha<1$. Then the weighted Bergman projection $P_{\gamma}$ maps $\Lambda_{\alpha}\left(D^{n}\right)$ onto $\Lambda_{\beta}\left(D^{n}\right)$, boundedly.

Proof: By (1.2), we have, by the same process as in the proof of Theorem 3.2,

$$
\begin{gathered}
\frac{\partial}{\partial z_{1}} P_{\gamma} f(z)=C_{n, \gamma} \int_{D^{n}} f(\zeta) \frac{(\gamma+2) \bar{\zeta}_{1}\left(1-\left|\zeta_{1}\right|^{2}\right)^{\gamma}}{\left(1-\bar{\zeta}_{1} z_{1}\right)^{\gamma+3}} \prod_{j=2}^{n} \frac{\left(1-\left|\zeta_{j}\right|^{2}\right)^{\gamma}}{\left(1-\overline{\zeta_{j}} z_{j}\right)^{\gamma+2}} d V(\zeta) \\
=C_{n, \gamma}(\gamma+2) \int_{D^{n}} \frac{\bar{\zeta}_{1}\left(f(\zeta)-f\left(z_{1}, \zeta_{2}, \cdots, \zeta_{n}\right)\right)\left(1-\left|\zeta_{1}\right|^{2}\right)^{\gamma}}{\left(1-\bar{\zeta}_{1} z_{1}\right)^{\gamma+3}} \\
\times \prod_{j=2}^{n} \frac{\left(1-\left|\zeta_{j}\right|^{2}\right)^{\gamma}}{\left(1-\overline{\zeta_{j}} z_{j}\right)^{\gamma+2}} d V(\zeta) .
\end{gathered}
$$

Then, by (i) and (ii) of Lemma 2.1, we have

$$
\begin{aligned}
\left|\frac{\partial}{\partial z_{1}} P_{\gamma} f(z)\right| & \lesssim|f|_{\Lambda_{\alpha}\left(D^{n}\right)} \int_{D^{n}} \frac{\left|\zeta_{1}-z_{1}\right|^{\alpha}\left(1-\left|\zeta_{1}\right|^{2}\right)^{\gamma}}{\left|1-\bar{\zeta}_{1} z_{1}\right|^{\gamma+3}} \prod_{j=2}^{n} \frac{\left(1-\left|\zeta_{j}\right|^{2}\right)^{\gamma}}{\mid 1-\overline{\left.\zeta_{j} z_{j}\right|^{\gamma+2}} d V(\zeta)} \\
& \lesssim|f|_{\Lambda_{\alpha}\left(D^{n}\right)} \frac{1}{\left(1-\left|z_{1}\right|^{2}\right)^{1-\alpha}} \prod_{j=2}^{n} \log \frac{1}{1-\left|z_{j}\right|^{2}}
\end{aligned}
$$

Let $0<\varepsilon<\alpha$. Then it follows that

$$
\begin{aligned}
\frac{1}{\left(1-\left|z_{1}\right|^{2}\right)^{1-\alpha}} \prod_{j=2}^{n} \log \frac{1}{1-\left|z_{j}\right|^{2}} & \lesssim \frac{1}{\min _{1 \leqslant j \leqslant n}\left(1-\left|z_{j}\right|^{2}\right)^{1-\alpha+\varepsilon}} \\
& \lesssim \frac{1}{\delta_{D^{n}(z)^{1-\alpha+\varepsilon}}}
\end{aligned}
$$

By (3.2) and (3.3), we have

$$
\left|P_{\gamma} f(z)\right| \lesssim|f|_{\Lambda_{\alpha}\left(D^{n}\right)} \frac{1}{\delta_{D^{n}(z)^{1-\alpha+\varepsilon}}}
$$

Thus we get the result.

\section{REFERENCES}

[1] H. Ahn and H.R. Cho, 'Optimal non-isotropic $L^{p}$ estimates with weights for the $\bar{\partial}$-problem on strictly pseudoconvex domains', Kyushu J. Math. (to appear).

[2] F. Beatrous, 'Estimates for derivatives of holomorphic functions in pseudoconvex domains', Math. Z. 191 (1986), 91-116. 
[3] H.R. Cho and J. Lee, 'Optimal weighted estimates for the Cauchy-Riemann equation on analytic polyhedra', Bull. Austral. Math. Soc. 59 (1999), 427-431.

[4] H.O. Kim and E.G. Kwon, 'Weighted subspaces of Hardy spaces', Canad. J. Math. 40 (1988), 1074-1083.

[5] S.G. Krantz, 'Lipschitz spaces, smoothness of functions, and approximation theory', Expo. Math. 3 (1983), 193-260.

[6] E.G. Kwon, 'Image area and the weighted subspaces of Hardy spaces', Canad. Math. Bull. 33 (1990), 167-174.

[7] W. Rudin, Function theory in the unit ball of $\mathbb{C}^{n}$ (Springer-Verlag, New York, 1980).

Department of Mathematics Education Andong National University

Andong 760-749

Korea

e-mail: chohr@andong.ac.kr
Department of Mathematics

Pusan National University

Pusan 609-735

Korea

e-mail: jklee235@pusan.ac.kr 